\title{
The Effects of High-Intensity Interval Training on Skeletal Muscle Morphological Changes and Denervation Gene Expression of Aged Rats
}

\author{
Seyed Morteza Tayebi ${ }^{1}$, Marefat Siahkouhian ${ }^{2}$, Mohammad Keshavarz $^{3}$, Mojgan Yousefi ${ }^{4}$ \\ Affiliations: 'Allameh Tabataba'i University, Faculty of Sport Science, Department of Exercise Physiology, Core Research \\ of Health Physiology and Physical Activity, Tehran, Iran, ${ }^{2}$ University of Mohaghegh Ardabili, Faculty of Educational Sci- \\ ence, Department of Exercise Science, Ardabil, Iran, ${ }^{3}$ Tarbiat Modares University, Faculty of Humanities, Department of \\ Exercise Science, Tehran, Iran, ${ }^{4}$ Avicenna Fertility Center, Department of Midwifery, Tehran, Iran
}

Correspondence: M. Siahkouhian, University of Mohaghegh Ardabili, Faculty of Educational Science, Office 232, Daneshgah St, Ardabil, Iran, e-mail: m_siahkohian@uma.ac.ir

ABSTRACT Muscle denervation is one of the most critical pathological factors involved in muscle atrophy as a result of ageing. This study aims to investigate the chronic effect of high-intensity interval training (HIIT) on cross-sectional areas and muscle denervation genes, interpreted in the plantaris muscle atrophy of aged rats. Twenty-eight aged and young rats were divided into four different groups, including exercise and control. The training protocol included six weeks of HIIT. Animals were sacrificed 48 hours after the last training session, and the plantaris muscle was removed. To measure Gadd45a mRNA and NCAM1mRNA, we used a real-time PCR technique. The cross-sectional area was measured with photomyography using an $\mathrm{H} \& \mathrm{E}$ technique. The results showed that ageing significantly decreased NCAM1 mRNA in the aged control group ( $\mathrm{p}=0.0001)$ and exercise leads to a significant increase (interaction effect) of it $(\mathrm{p}=0.003)$. Gadd45a mRNA was significantly increased due to ageing $(\mathrm{p}=0.009)$, and exercise resulted in a significant decrease in that in the aged exercise group $(\mathrm{p}=0.04)$. The cross-sectional area in the aged control group was significantly smaller than in the young control group $(p=0.046)$. In contrast to young groups, exercise could increase the cross-sectional area in the aged exercise group compared with the aged control group, but it was not significant $(\mathrm{p}=0.069)$. It seems that HIIT could improve ageing-induced muscular atrophy, although denervation-involved gene modification leads to an increase in the muscular cross-sectional area; therefore, it improves muscular atrophy due to ageing.

KEY WORDS high-intensity interval training, muscular atrophy, muscle denervation, ageing, gadd45a protein, ncam1 protein

$@$ MJSSMontenegro

HIIT EFFECTS ON DENERVATION IN THE SKELETAL MUSCLE OF AGED RATS

http://mjssm.me/?sekcija=article\&artid=181

\section{Introduction}

Ageing deteriorates muscle strength and mass (known by the term "sarcopenia"). This leads to a reduced functional capacity as well as an increased risk of chronic metabolic diseases (Kurokawa, Mimori, Tanaka, Kohriyama, \& Nakamura, 1999). Sarcopenia can be found particularly in muscle fibre research shows that fast twitch fibres are most affected as a result of ageing (Snijders, Verdijk, \& van Loon, 2009). Diminished numbers of motoneurons due to ageing could reduce the number and size of muscle fibres. This can cause impaired muscle functions, such as maximum power, power, and rate of force development (RFD), which play significant roles in routine activities (Aagaard, Suetta, Caserotti, Magnusson, \& Kjaer, 2010). Evidence suggests that motoneuron failure is one of the causes of sarcopenia, and this is because of its connection to muscle fibres. Therefore, the loss of motoneurons is the first incidence in fast twitch muscles damage during ageing (Drey et al., 2014). However, the number and size of motoneurons do not change in the spinal cord during the whole life, but this does happen in neuromuscular junctions (Chai, Vukovic, Dunlop, Grounds, \& Shavlakadze, 2011). During ageing, motoneurons' capability is subjected to be shrunk, and muscle fibres

Received: January 182019 | Accepted after revision: March 212019 | First published online: September 012019

(C) 2019 by the author(s). License MSA, Podgorica, Montenegro. This article is an open access article distributed under the terms and conditions of the Creative Commons Attribution (CC BY).

Conflict of interest: None declared. 
and motoneurons connections would become thinner; this ultimately leads to muscular atrophy (Gyorkos \& Spitsbergen, 2014). Therefore, recognizing the mechanisms involved in neuromuscular degeneration, and the interventions that can reverse these mechanisms is essential.

There is strong evidence showing that, after denervation, acetylcholine receptors, as well as NCAM1 (Knudsen, McElwee, \& Myers, 1990; Walsh, Hobbs, Wells, Slater, \& Fazeli, 2000) and Gadd45a, which induce atrophy (Barns et al., 2014; Bongers et al., 2013), are increased. In contrast, it has been shown that exercise not only create a non-surgical and non-pharmacological protective mechanism against diseases and musculoskeletal disabilities, but it is also assumed to be a way of maintaining the function and structure of synapses and regenerating damaged neurons (Gyorkos \& Spitsbergen, 2014; Smith \& Mulligan, 2014). In addition to endurance/aerobic training (Samiei, Behpour, Tadibi, \& Fathi, 2018), studies show HIIT exercises have many benefits, including improving glucose levels in diabetic subjects (Rahmaty, Gaeini, Dolatshahi, \& Choobineh, 2018), reducing inflammation in adipose tissue (Alizadeh, Asad, Faramarzi, \& Afroundeh, 2017), and increasing prolonged motoneuron maintenance and neuromuscular junctions (M. R. Deschenes et al., 1993) and has led to neuronal sprouting and proliferation in both healthy and unhealthy subjects (Sabatier, Redmon, Schwartz, \& English, 2008). Therefore, the purpose of this study is to investigate the effect of six weeks of HIIT on NCAM1 and Gadd45a gene expression in order to observe denervation in the plantaris muscle of aged rats. In addition, because the role of exercise to modify these factors in the neuromuscular junction has not been studied yet, and in order to ensure that NCAM1 and Gadd45a are reliable factors, the effect of exercise on changes in the muscular cross-sectional area will also be identified.

\section{Methods}

Animals

Experiment protocols for rats were planned according to the policies of the Iranian Convention for the Protection of Vertebrate Animals, and the Ethics Committee of the School of Medicine Sciences, Tarbiat Modares University (TMU), authorized the protocol. Twenty-four male Wistar rats (aged 4-5 months), obtained from Iran Pasteur Institute were collected in the current study. They dwelled in a standard temperature room and 12-h light and dark periods with unlimited access to water and food. They were maintained in the Animal House in the School of Medical Sciences of TMU. Animals (5 in each group) were randomly divided into four groups: Aged Control (AC) Aged Training (AT), Young Control (YC), and Young Training (YT).

\section{Training protocol}

The training groups performed five sessions each week of HIIT for six weeks, and 48 hours after the last training session, the training groups did not perform any exercise until they were sacrificed. After the animals were sacrificed, the left foot plantaris muscle was frozen immediately in liquid nitrogen for cellular and molecular testing, and then stored in a refrigerator for 80 days. The right leg muscle was used for conducting a 10-25\% formalin test for histochemical experiments. In the current study, the plantaris muscle was chosen because it is predominantly composed of (II-60\%) muscle fibres of type II (Michael R Deschenes, Roby, \& Glass, 2011).

At the beginning, in order to reduce stress as well as create familiarity with running on a treadmill, the subjects ran on a training programme for a week at speeds of 10 to 18 metres per minute $(\mathrm{m} / \mathrm{min})$ for ten minutes. Then, 48 hours after the last familiarization session, an exhausting test was performed, starting at a speed of $10 \mathrm{~m} / \mathrm{min}$ and increased by $3 \mathrm{~m}$ for every three minutes (Thomas, Bishop, Moore-Morris, \& Mercier, 2007).

\begin{tabular}{|c|c|c|c|c|c|c|c|}
\hline Week & Sets & $\begin{array}{c}\text { Rest \& } \\
\text { Exercise } \\
\text { Ratio } \\
\end{array}$ & $\begin{array}{l}\text { Exercise } \\
\text { Intensity }\end{array}$ & $\begin{array}{c}\text { Rest } \\
\text { Intensity }\end{array}$ & $\begin{array}{c}\text { Average } \\
\text { Speed in } \\
\text { Week }\end{array}$ & $\begin{array}{c}\text { Time of } \\
\text { Training } \\
\text { (minutes) }\end{array}$ & $\begin{array}{c}\text { Days } \\
\text { per } \\
\text { week }\end{array}$ \\
\hline 1 & 5 & $2: 2$ & $80 \%$ & $60 \%$ & $70 \%$ & 90 & 5 \\
\hline 2 & 6 & $2: 2$ & $80 \%$ & $60 \%$ & $70 \%$ & 110 & 5 \\
\hline 3 & 7 & $2: 2$ & $90 \%$ & $50 \%$ & $70 \%$ & 130 & 5 \\
\hline 4 & 8 & $2: 2$ & $100 \%$ & $50 \%$ & $75 \%$ & 150 & 5 \\
\hline 5 & 8 & $2: 2$ & $100 \%$ & $50 \%$ & $75 \%$ & 150 & 5 \\
\hline 6 & 8 & $2: 2$ & $100 \%$ & $50 \%$ & $75 \%$ & 150 & 5 \\
\hline
\end{tabular}

The time to fatigue was determined by the inability of the rats to run on the treadmill. After determining the maximum speed, the training groups participated in a six-week HIIT programme (Tables 1 and 2).

\begin{tabular}{|c|c|c|c|c|c|}
\hline Groups & Speed level & Week $1^{\text {st }} \& 2^{\text {nd }}$ & Week $3^{\text {rd }}$ & Week $4^{\text {th }}$ & Week $6^{\text {th }} \& 7^{\text {th }}$ \\
\hline \multirow{3}{*}{ YT } & Max Speed & 40 & 44 & 44 & 47 \\
\hline & Sprint Interval & 32 & 40 & 44 & 47 \\
\hline & Rest Interval & 24 & 22 & 22 & 24 \\
\hline \multirow{3}{*}{ AT } & Max Speed & 34 & 37 & 37 & 40 \\
\hline & Sprint Interval & 27 & 33 & 37 & 40 \\
\hline & Rest Interval & 20 & 19 & 19 & 40 \\
\hline
\end{tabular}

Note. YT: Young Training; AC: Aged Control; AT: Aged Training. 
Moulding and cutting of muscle

Forty-eight hours after fixing the tissues in formalin, the samples were distilled from alcohol into different degrees of purity (70,80,90, and 100), the stopping time of each one in alcohol containers was calculated for two hours. In the next step, a microplate was used to cut them, and 5 microns of each sample were placed in the required measurements. The slides then were placed in a hot bath for at least $60^{\circ} \mathrm{C}$ to melt the paraffin from the tissue section; in addition to this, the tissue was stuck to the lamina. Removed sections were then transferred to the slide, and after removing paraffin in an oven at $120^{\circ}$ for 10 minutes and washing steps in alcohol of different purities, immunohistochemical staining and $\mathrm{H} \& \mathrm{E}$ were performed.

$H$ \& E staining and cross-sectional measurements

To measure the cross-sectional area (CSA) of the muscle, muscle samples were fixed in $10 \%$ formalin and then moulded into paraffin. The fixed organ tissues were then embedded in paraffin. The paraffinized tissue specimens were cut into 5 um-thick slices and were stained with hematoxylin and eosin (H\&E) for histopathological observation using a previously established procedure (Carleton, Drury, \& Wallington, 1967). Photomicrographs were captured using a normal spectra fluorescent microscope (Olympus DP 72) at 6100× magnification with an attached digital camera (Olympus, Tokyo, Japan).

\section{Real-time PCR}

Total RNA was extracted from the plantaris muscle samples by exerting QIAzol ${ }^{\circ}$ Lysis Reagent (Qiagen) according to the manufacturer's recommendations. RNA concentrations were defined by the rate of absorbance at $260 \mathrm{~nm}$. RNA purity also was determined by absorbance ratio at 260 and $280 \mathrm{~nm}$, and with a Nano-Drop Machine. Acceptable purification in $260 / 280 \mathrm{~nm}$ absorbance ratio above 1.8. RNA was reverse-transcribed into complementary DNA (cDNA) using a Revert Aid first standard cDNA Synthesis Kit (Thermo Scientific, Fermentas K1622, United States) using an accepted protocol including reverse transcription at $25^{\circ} \mathrm{C}$ for five minutes, then inactivated reverse transcriptase at $42^{\circ} \mathrm{C}$ for 60 minutes, and finally refrigeration at $70{ }^{\circ} \mathrm{C}$ for five minutes, with storage at $-20^{\circ} \mathrm{C}$.

For real-time PCR, primers were designed using NCBI and gene runner software and synthesized by Cinnagen Company (Iran). The primer sequences are shown in Table 3. Gene expression measurement was done with Master Mix and SYBR Green in an Applied Biosystems, StepOne $e^{\text {tix }}$ thermal cycler. The thermal cycle protocol was divided into protocols including one cycle at $95^{\circ} \mathrm{C}$ in $10 \mathrm{~min}$, followed by 40 cycles at $95^{\circ} \mathrm{C}$ for $15 \mathrm{~s}$, and $60^{\circ} \mathrm{C}$ for $30 \mathrm{~s}$. PCR amplification also was performed with duplication in a total reaction volume in $20 \mu \mathrm{l}$. The reaction mixture had three $\mu$ l diluted templates, $10 \mu \mathrm{l}$ SYBR Premix Ex Taq $q^{\text {Tw }}$ Kit (Perfect Real Time, Takara Code RR041A, Japan), and two $\mu$ l primers. Amplification specificity was monitored using the analysis of the melting curve. Genes' relative expression were normalized by subtracting the housekeeping levels of the mean of glyceraldehyde 3-phosphate dehydrogenase (Gapdh) 2- $\Delta \Delta \mathrm{CT}$, which was amplified as a housekeeping gene. All data are represented as fold changes from the weight-bearing group (Livak \& Schmittgen, 2001).

\begin{tabular}{cl} 
TABLE 3 Sequences of the primer designed in this study \\
Primer & sequences \\
\hline NCAM1 & $\begin{array}{l}\text { forward 5'CCAGTACATTGAGCACCTAC3' } \\
\text { reverse 5'GCAAATGATCTACTGGGTTG3' } \\
\text { Gadd4a } \\
\text { forward 5'TAACTGTCGGGTGTACGAG3 } \\
\text { reverse 5'GCAACAGAAAGCACGAATGA3' } \\
\text { forward 5'GACATGCCGCCTGGAGAAA3' } \\
\text { reverse 5'AGCCAGGATGCCCTTTAGT3' }\end{array}$ \\
\hline
\end{tabular}

Statistical Analysis

All statistical analyses were done using SPSS software (version 20, SPSS Inc., Chicago, IL, USA). The normal assumption was examined using a one-sample Kolmogorov-Smirnov test. Two-way ANOVA tests were used to compare groups regarding under study variables, and the significant level was determined at $\mathrm{p}<0.05$.

Results

Body mass

Changes in body mass before and after exercise are shown in Table 3 . The results of the t-test for intra-group comparison of body mass changes showed that the weight of both young groups increased significantly at the end of six weeks compared to the baseline measurements $(p=0.001)$. However, the control group and the aged training group showed a non-significant increase compared to the pre-test data $(\mathrm{p}=0.655, \mathrm{p}=0.83$, respectively) (Table 4).

TABLE 4 Weight changes in the experimental groups

\begin{tabular}{cccc} 
Variable & Group & Pre - test & Post - test \\
\hline & YC & $314.15 \pm 17.63$ & $370.23 \pm 18.52^{*}$ \\
Body Mass & YT & $299.82 \pm 10.54$ & $347.03 \pm 17.15^{*}$ \\
& AC & $386.02 \pm 9.55$ & $390.37 \pm 55.09$ \\
& AT & $407.23 \pm 11.51$ & $400.35 \pm 1.76$ \\
\hline
\end{tabular}

Note. YC: Young Contro; YT: Young Training; AC: Aged Control; AT: Aged Training; *: Significant Difference to pre-test $(\mathrm{p}=0.001)$. 
Plantaris muscle weight

The results of one-way ANOVA to compare absolute weight and relative weight (muscle/body mass ratio) of the plantaris muscle showed a significant difference between groups $(\mathrm{p}=0.001 ; \mathrm{p}=0.001)$. Therefore, Tukey's post hoc test showed that the absolute and relative weight of the plantaris muscle in aged rats was significantly lower than young groups ( $\mathrm{p}=0.002, \mathrm{p}=0.001$ ) (Table 5 ). HIIT significantly decreased muscle weight in young rats $(\mathrm{P}=0.99)$, but the relative ratio was increased, although the difference was not statistically significant between these two groups $(\mathrm{p}=0.466)$. In comparison with the young groups, HIIT increased both muscle weight $(\mathrm{p}=0.106)$ and relative weight $(\mathrm{p}=0.425)$ in the aged training group.

\begin{tabular}{ccccc}
\multicolumn{5}{l}{ TABLE 5 The average of relative and absolute plantaris weight } \\
Variable & YC & YT & AC & AT \\
\hline Plantaris Weight $(\mathrm{mg})$ & $0.3949 \pm 0.024$ & $0.378 \pm 0.033$ & $0.321 \pm 0.048^{*}$ & $0.364 \pm 0.039$ \\
Relative Weight $(\mathrm{mg} / \mathrm{g})$ & $0.00106 \pm 0.0007$ & $0.00115 \pm 0.000078$ & $0.000083 \pm 0.00016$ & $0.0009 \pm 0.0007$ \\
\hline
\end{tabular}

Note. YC: Young Control; YT: Young Training; AC: Aged Control; AT: Aged Training; *: Significant Difference to YT ( $p<0.05)$.

\section{Cross section area (CSA) of muscle fibres}

The results of one-way analysis of variance analysis showed a significant difference between groups $(\mathrm{P}=0.032)$. Tukey's post hoc test showed that the cross-sectional area of the muscle fibres in the control aged group was significantly less than in young groups $(\mathrm{P}=0.046)$ (Figure $1 \mathrm{~A}$ and $\mathrm{C})$. Compared to the young groups, there was a non-significant decrease in the cross-sectional area of the trained rats as opposed to the control $(\mathrm{P}=0.53)$ (Figure $1-\mathrm{A}$ and $-\mathrm{B}$ ).

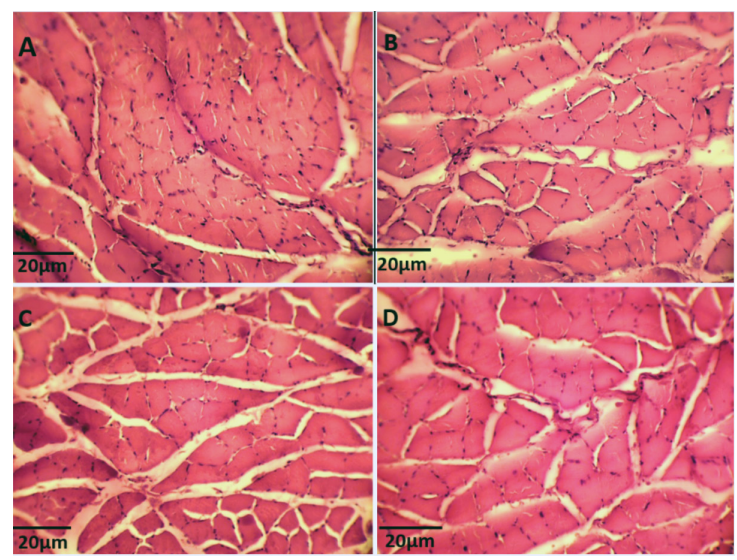

FIGURE 1 Plantaris Micrograph Photo. (A): Young Control; (B): Young Training; (C): Aged Control; (D): Aged Training.

Unlike with the young groups, exercise significantly increased the size of muscle fibres in the aged training group $(\mathrm{P}=0.69)$ (Fig. $1-\mathrm{C}$ and $-\mathrm{D})$, but still less than in the young groups according to the data (Table 6).

\begin{tabular}{ccccc}
\multicolumn{5}{l}{ TABLE 6 Cross section area (CSA) of muscle fibres } \\
Variable & YC & YT & AC & AT \\
\hline CSA $(\mu \mathrm{m})$ & $308.47 \pm 60.32$ & $297.1 \pm 46.94$ & $260.46 \pm 60.32$ & $273.74 \pm 31.04$ \\
\hline
\end{tabular}

Note. CSA: Cross Section Area; YC: Young Control; YT: Young Training; AC: Aged Control; AT: Aged Training; *: Significant Difference to $Y C(p<0.05)$.

\section{mRNA expression of Gadd45a}

The results show that HIIT has significantly reduced Gadd45a expression in the plantaris muscle of aged rats $(p=0.041)$. Ageing resulted in a significant increase in this gene $(p=0.0001)$, but in the young group, exercise did not show a significant effect on Gadd45a expression ( $\mathrm{p}=0.465)$ (Figure 2).

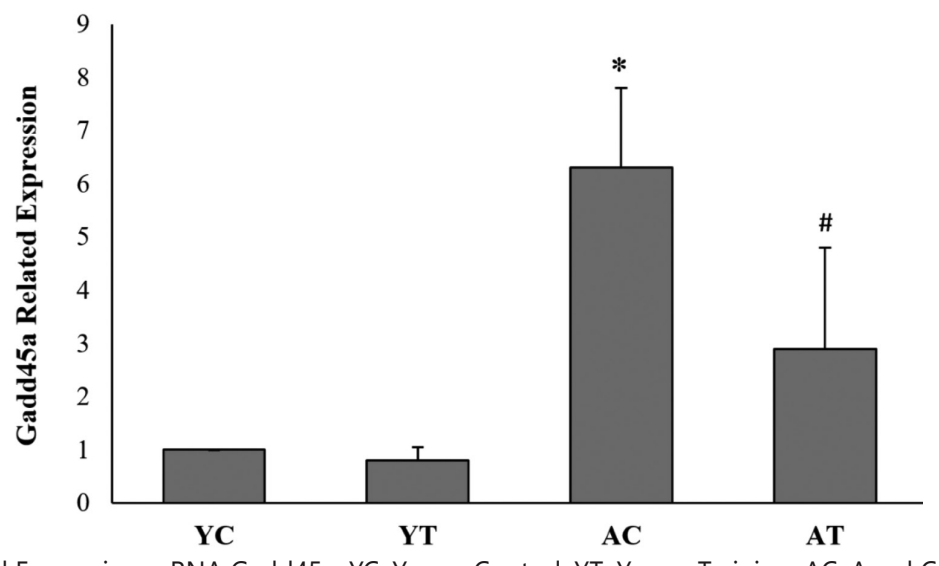

FIGURE 2 Related Expression mRNA Gadd45a. YC: Young Control; YT: Young Training; AC: Aged Control; AT: Aged Training; *: Significant Difference to YC. \#: Significant Difference to AC. 
mRNA expression of NCAM1

In contrast, HIIT significantly increased NCAM1 mRNA expression in the plantaris muscle of aged rats (P $=0.001)$. Moreover, ageing resulted in a significant decrease in this gene $(\mathrm{P}=0.0040)$, but in the YT group, exercise did not show a significant effect on NCAM1 mRNA expression $(\mathrm{P}=0.315)$ (Figure 3).

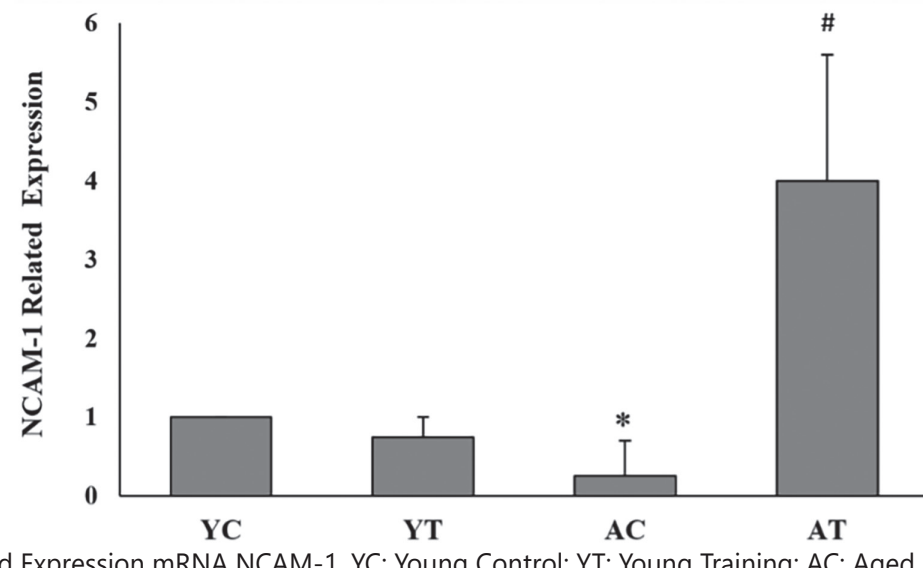

FIGURE 3 Related Expression mRNA NCAM-1. YC: Young Control; YT: Young Training; AC: Aged Control; AT: Aged Training; *: Significant Difference to YC. \#: Significant Difference to AC.

\section{Discussion}

The findings have shown that consistent with previous research results, ageing significantly reduced the weight of the plantaris muscle and diminished the cross section area and relative muscle weight. In contrast, the results have shown that six weeks of HIIT almost reduced the size of the plantaris muscle in young rats. This reduction in muscle fibres after exercise could be advantageous because it has been reported that change of muscle fibres size after endurance training reduces the distance between muscle fibres and blood capillaries, where gas exchange should occur (Hoppeler, Howald, \& Cerretelli, 1990). Unlike young rats, the aged group showed an increase in muscle weight and size after training. This difference between the aged and young groups muscles' response to exercise can be due to differences in their compatibility capacity. Since ageing causes atrophy in muscle fibres, this kind of muscle fibres has a higher potential for hypertrophy, even in response to endurance exercises that usually do not lead to hypertrophy (Michael R Deschenes, Hurst, Ramser, \& Sherman, 2013). To support this claim, reference can be made to Cogan's study, in which it was reported that endurance training leads to muscle fibroblastic hypertrophy in human subjects (Coggan et al., 1992).

During embryonic growth, acetylcholine receptors are expressed with fetal isoforms by muscle fibres before becoming neurons. The high volume of receptors makes fibres extremely sensitive to acetylcholine. After neurotization, a significant decrease in acetylcholine receptors expression occurs except for the muscular cells' area, which is assumed to be a communication site between nerves and muscle (Cohen et al., 2007). Loss of the nervous system is concurrent with the expression of all subtypes of acetylcholine receptors. In addition, it over-expresses Gadd45 subcategories.

Regarding this evidence, our results showed that there was a significant increase in Gadd45a expression in aged plantaris muscle compared to young muscle. Gadd45a also shows an almost twofold increase from 15 to 24 months and then a decrement with age (Barns et al., 2014). Studies have shown that the nervous system's response to damage caused by peripheral nerve suppression is different in healthy rats and NCAM-free mice; therefore, peripheral axons of motor neurons are reshaped after surgical abnormalities in normal healthy rats, but axon growth is impaired and defective in NCAM-free mice (Grumbles, Almeida, \& Thomas, 2008). A study done on surgically debilitated young and aged mice showed that a muscle twitch after nerve rupture in young mice resulted in a 33\% increase in NCAM (Biral et al., 2008).

In comparison to aged rats, axonal growth defects are associated with no significant change in NCAM; this could be attributed to the inability to regenerate peripheral nerves in aged subjects in the absence of NCAM changes (Gatchalian, Schachner, \& Sanes, 1989). In addition, physical activity as a non-pharmacological intervention has great importance in the function and structure maintenance of synapses and recovering damaged neurons. Studies that examine the effect of exercise on muscle show that endurance exercise improves the reabsorbed muscle fibres (English, Cucoranu, Mulligan, \& Sabatier, 2009; Krakowiak et al., 2015) and the number of myelin axons after peripheral nerve damage (Asensio-Pinilla, Udina, Jaramillo, \& Navarro, 2009; Bobinski, Martins, Bratti, \& Mazzardo-Martins, 2011). HIIT also increases neuromuscular stability and prolongs neuronal growth more than low-intensity exercises do (M. R. Deschenes et al., 1993; Sabatier et al., 2008). The amount of muscular activity directly affects NMJ size (M. R. Deschenes et al., 1993): it can be concluded that the amount of involved muscle during an exercise activity significantly influences NMJ structures. For example, it has been shown that in comparison to plantaris and EDL muscles, followed by exercise activity, the changes only applied to the plantaris muscle (Alshuaib \& Fahim, 1990). 
In conclusion, the results of this study revealed an increased Gadd45a level and a decreased level of NCAM1 in the plantaris muscles of aged rats, which was associated with a decrease in the muscle's cross-sectional area. Therefore, we can argue that as the process of sarcopenia develops from type 2 or fast muscle fibres, there is a relationship between the amount of muscle fibres and musculoskeletal system health. In this regard, the benefits of higher intensity exercises to various physical systems in different populations, such as athletes, patients, and the elderly, are well-defined (Madden, 2018; Ramírez-Vélez et al., 2016). Elderly people also may benefit from high-intensity exercises to improve their musculoskeletal health, in order to reduce the effects of ageing on muscle mass, and ultimately prevent their disabilities in doing routine activities and preventing metabolic diseases.

\section{RE FERENCES}

Aagaard, P., Suetta, C., Caserotti, P., Magnusson, S. P., \& Kjaer, M. (2010). Role of the nervous system in sarcopenia and muscle atrophy with aging: strength training as a countermeasure. Scandinavian Journal of Medicine and Science in Sports, 20(1), 49-64. doi: 10.1111/j.1600-0838.2009.01084.x

Alizadeh, M., Asad, M. R., Faramarzi, M., \& Afroundeh, R. (2017). Effect of Eight-Week High Intensity Interval Training on Omentin-1 Gene Expression and Insulin-Resistance in Diabetic Male Rats. Annals of Applied Sport Science, 5(2), 29-36. doi: 10.18869/acadpub.aassjournal.5.2.29

Alshuaib, W. B., \& Fahim, M. A. (1990). Effect of exercise on physiological age-related change at mouse neuromuscular junctions. Neurobiology of aging, 11(5), 555-561. doi: 10.1016/0197-4580(90)90117-I

Asensio-Pinilla, E., Udina, E., Jaramillo, J., \& Navarro, X. (2009). Electrical stimulation combined with exercise increase axonal regeneration after peripheral nerve injury. Brain Stimulation in Psychiatry, 219(1). doi: 10.1016/j.expneurol.2009.05.034

Barns, M., Gondro, C., Tellam, R. L., Radley-Crabb, H. G., Grounds, M. D., \& Shavlakadze, T. (2014). Molecular analyses provide insight into mechanisms underlying sarcopenia and myofiber denervation in old skeletal muscles of mice. The international journal of biochemistry \& cell biology, 53, 174-185. doi: 10.1016/j.biocel.2014.04.025

Biral, D., Kern, H., Adami, N., Boncompagni, S., Protasi, F., \& Carraro, U. (2008). Atrophy-resistant fibers in permanent peripheral denervation of human skeletal muscle. Neurological research, 30(2), 137-144. doi: $10.1179 / 174313208 \times 281145$

Bobinski, F., Martins, D. F., Bratti, T., \& Mazzardo-Martins, L. (2011). Neuroprotective and neuroregenerative effects of low-intensity aerobic exercise on sciatic nerve crush injury in mice. Neuroscience, 194. doi: 10.1016/j.neuroscience.2011.07.075

Bongers, K. S., Fox, D. K., Ebert, S. M., Kunkel, S. D., Dyle, M. C., Bullard, S. A., . . Adams, C. M. (2013). Skeletal muscle denervation causes skeletal muscle atrophy through a pathway that involves both Gadd45a and HDAC4. American Journal of Physiology-Endocrinology and Metabolism, 305(7), E907-E915. doi: 10.1152/ajpendo.00380.2013

Carleton, H. M., Drury, R. A. B., \& Wallington, E. A. (1967). Carleton's Histological Technique. Oxford: Oxford University Press.

Chai, R. J., Vukovic, J., Dunlop, S., Grounds, M. D., \& Shavlakadze, T. (2011). Striking denervation of neuromuscular junctions without lumbar motoneuron loss in geriatric mouse muscle. PloS one, 6(12), e28090. doi: 10.1371/journal.pone.0028090

Coggan, A. R., Spina, R. J., King, D. S., Rogers, M. A., Brown, M., Nemeth, P. M., \& Holloszy, J. O. (1992). Skeletal muscle adaptations to endurance training in 60-to 70-yr-old men and women. Journal of Applied Physiology, 72(5), 1780-1786. doi: 10.1152/jappl.1992.72.5.1780

Cohen, T. J., Waddell, D. S., Barrientos, T., Lu, Z., Feng, G., Cox, G. A., . . Yao, T.-P. (2007). The histone deacetylase HDAC4 connects neural activity to muscle transcriptional reprogramming. Journal of Biological Chemistry, 282(46), 33752-33759. doi: 10.1074/jbc.M706268200

Deschenes, M. R., Hurst, T. E., Ramser, A. E., \& Sherman, E. G. (2013). Presynaptic to postsynaptic relationships of the neuromuscular junction are held constant across age and muscle fiber type. Developmental neurobiology, 73(10), 744-753. doi: 10.1002/dneu.22095

Deschenes, M. R., Maresh, C. M., Crivello, J. F., Armstrong, L. E., Kraemer, W. J., \& Covault, J. (1993). The effects of exercise training of different intensities on neuromuscular junction morphology. Journal of Neurocytology, 22, 603-615. doi: 10.1007/BF01181487

Deschenes, M. R., Roby, M. A., \& Glass, E. K. (2011). Aging influences adaptations of the neuromuscular junction to endurance training. Neuroscience, 190, 56-66. doi: 10.1016/j.neuroscience.2011.05.070

Drey, M., Krieger, B., Sieber, C. C., Bauer, J. M., Hettwer, S., Bertsch, T., .. S Schuster, G. (2014). Motoneuron loss is associated with sarcopenia. Journal of the American Medical Directors Association, 15(6), 435-439. doi: 10.1016/j.jamda.2014.02.002

English, A. W., Cucoranu, D., Mulligan, A., \& Sabatier, M. (2009). Treadmill training enhances axon regeneration in injured mouse peripheral nerves without increased loss of topographic specificity. The Journal of comparative neurology, 517(2), 245-255. doi: 10.1002/cne.22149

Gatchalian, C. L., Schachner, M., \& Sanes, J. R. (1989). Fibroblasts that proliferate near denervated synaptic sites in skeletal muscle synthesize the adhesive molecules tenascin (J1), N-CAM, fibronectin, and a heparan sulfate proteoglycan. The Journal of cell biology, 108(5), 1873-1890. doi: 10.1083/ jcb.108.5.1873 
Grumbles, R. M., Almeida, V. W., \& Thomas, C. K. (2008). Embryonic neurons transplanted into the tibial nerve reinnervate muscle and reduce atrophy but NCAM expression persists. Neurological research, 30(2), 183-189. doi: 10.1179/174313208X281073

Gyorkos, A. M., \& Spitsbergen, J. M. (2014). GDNF content and NMJ morphology are altered in recruited muscles following high-speed and resistance wheel training. Physiological reports, 2(2), e00235. doi: 10.1002 /phy2.235

Hoppeler, H., Howald, H., \& Cerretelli, P. (1990). Human muscle structure after exposure to extreme altitude. Experientia, 46(11-12), 1185-1187. doi: 10.1007/BF01936933

Knudsen, K. A., McElwee, S. A., \& Myers, L. (1990). A role for the neural cell adhesion molecule, NCAM, in myoblast interaction during myogenesis. Developmental biology, 138(1), 159-168. doi: 10.1016/00121606(90)90185-L

Krakowiak, J., Liu, C., Papudesu, C., Ward, P. J., Wilhelm, J. C., \& English, A. W. (2015). Neuronal BDNF Signaling Is Necessary for the Effects of Treadmill Exercise on Synaptic Stripping of Axotomized Motoneurons. Neural Plasticity, 2015. doi: 10.1155/2015/392591

Kurokawa, K., Mimori, Y., Tanaka, E., Kohriyama, T., \& Nakamura, S. (1999). Age-related change in peripheral nerve conduction: compound muscle action potential duration and dispersion. Gerontology, 45(3), 168 173. doi: $10.1159 / 000022081$

Livak, K. J., \& Schmittgen, T. D. (2001). Analysis of relative gene expression data using real-time quantitative PCR and the 2- $\triangle \Delta$ CT method. Methods, 25(4), 402-408. doi: 10.1006/meth.2001.1262

Madden, A. (2018). The Efficacy of HIIT and HIRT in Older Adults. Unpublished Master Dissertation. Laramie, WY: University of Wyoming.

Rahmaty, S., Gaeini, A. A., Dolatshahi, M., \& Choobineh, S. (2018). The Effect of High-Intensity Interval Training and Continuous Training on the Desnutrin Gene Expression in the Subcutaneous Adipose Tissue and the Quadriceps Femoris Muscle Tissue of Obese Male Rats. Annals of Applied Sport Science, 6(2), 37-44. doi: 10.29252/aassjournal.6.2.37

Ramírez-Vélez, R., Hernandez, A., Castro, K., Tordecilla-Sanders, A., González-Ruíz, K., Correa-Bautista, J. E., . . García-Hermoso, A. (2016). High intensity interval-vs resistance or combined-training for improving cardiometabolic health in overweight adults (cardiometabolic hiit-rt study): study protocol for a randomised controlled trial. Trials, 17(1), 298. doi: 10.1186/s13063-016-1422-1

Sabatier, M. J., Redmon, N., Schwartz, G., \& English, A. W. (2008). Treadmill training promotes axon regeneration in injured peripheral nerves. Experimental Neurology, 211(2), 489-493. doi: 10.1016/j.expneurol.2008.02.013

Samiei, A., Behpour, N., Tadibi, V., Fathi, R. (2018). Effect of Eight Weeks of Aerobic Training on Some Myocardial Fibrosis Indices in Cardiac Muscle of Diabetic Rats. Annals of Applied Sport Science, 6(4), 1-8. doi: 10.29252/aassjournal.6.4.1

Smith, M. B., \& Mulligan, N. (2014). Peripheral neuropathies and exercise. Topics in Geriatric Rehabilitation, 30(2), 131-147. doi: 10.1097/TGR.0000000000000013

Snijders, T., Verdijk, L. B., \& van Loon, L. J. (2009). The impact of sarcopenia and exercise training on skeletal muscle satellite cells. Aging research reviews, 8(4), 328-338. doi: 10.1016/j.arr.2009.05.003

Thomas, C., Bishop, D., Moore-Morris, T., \& Mercier, J. (2007). Effects of high-intensity training on MCT1, MCT4, and NBC expressions in rat skeletal muscles: influence of chronic metabolic alkalosis. American Journal of Physiology-Endocrinology and Metabolism, 293(4), E916-922. doi: 10.1152/ajpendo.00164.2007

Walsh, F. S., Hobbs, C., Wells, D. J., Slater, C. R., \& Fazeli, S. (2000). Ectopic expression of NCAM in skeletal muscle of transgenic mice results in terminal sprouting at the neuromuscular junction and altered structure but not function. Molecular and Cellular Neuroscience, 15(3), 244-261. doi: 10.1006/mcne.1999.0815 